\title{
The Shi arrangements and the Bernoulli polynomials
}

\author{
Daisuke Suyama $^{*}$ Hiroaki Terao ${ }^{\dagger}$ \\ Department of Mathematics, Hokkaido University, Sapporo, Hokkaido 060-0810, Japan.
}

\begin{abstract}
The braid arrangement is the Coxeter arrangement of the type $A_{\ell}$. The Shi arrangement is an affine arrangement of hyperplanes consisting of the hyperplanes of the braid arrangement and their parallel translations. In this paper, we give an explicit basis construction for the derivation module of the cone over the Shi arrangement. The essential ingredient of our recipe is the Bernoulli polynomials.
\end{abstract}

Keywords: Hyperplane arrangement; Shi arrangement; Free arrangement; Derivations; Bernoulli polynomial

\section{Introduction}

Let $E$ be an $\ell$-dimensional Euclidean space and $\Phi$ be the root system of the type $A_{\ell}$. Let $\Phi_{+}$denote the set of positive roots. In this paper we explicitly choose $E$ and $\Phi$ as follows: let $W=\mathbb{R}^{\ell+1}$ and $x_{1}, \ldots, x_{\ell+1}$ be an orthonormal basis for the dual space $W^{*}$. Define

$$
\begin{aligned}
E & :=\left\{\sum_{i=1}^{\ell+1} c_{i} x_{i} \in W^{*} \mid \sum_{i=1}^{\ell+1} c_{i}=0\right\}, \\
\Phi & :=\left\{x_{i}-x_{j} \in E \mid 1 \leq i \leq \ell+1,1 \leq j \leq \ell+1, i \neq j\right\}, \\
\Phi_{+} & :=\left\{x_{i}-x_{j} \in \Phi \mid i<j\right\} .
\end{aligned}
$$

*email: s093014@math.sci.hokudai.ac.jp

${ }^{\dagger}$ Corresponding author. Supported by JSPS Grants-in-Aid, Scientific Research (B) No. 21340001. email: terao@math.sci.hokudai.ac.jp 
Let $\mathcal{A}(\Phi)=\left\{H_{\alpha} \mid \alpha \in \Phi_{+}\right\}$, where $H_{\alpha}=\{v \in W \mid \alpha(v)=0\}$. Then $\mathcal{A}(\Phi)$ is called a braid arrangement, which is undoubtedly the most-studied arrangement of hyperplanes in various contexts. In the study of the KazhdanLusztig representation theory of the affine Weyl groups, J.-Y. Shi introduced the Shi arrangements in [7] as follows: Let

$$
H_{\alpha, 1}=\{v \in W \mid \alpha(v)=1\} .
$$

Then the Shi arrangement is given by

$$
\mathcal{A}(\Phi) \cup\left\{H_{\alpha, 1} \mid \alpha \in \Phi_{+}\right\}=\bigcup_{\alpha \in \Phi_{+}}\left\{H_{\alpha}, H_{\alpha, 1}\right\}
$$

Embed the $(\ell+1)$-dimensional space $W$ into $V=\mathbb{R}^{\ell+2}$ by adding a new coordinate $z$ such that $W$ is defined by the equation $z=1$ in $V$. Then, as in [5, Definition 1.15], we have the cone $\mathcal{S}_{\ell}$ over the Shi arrangement. It is a central arrangement in $V$ defined by

$$
Q\left(\mathcal{S}_{\ell}\right)=z \prod_{1 \leq p<q \leq \ell+1}\left(x_{p}-x_{q}\right) \prod_{1 \leq p<q \leq \ell+1}\left(x_{p}-x_{q}-z\right)=0 .
$$

Let $S$ be the algebra of polynomial functions on $V$ and let $\operatorname{Der}_{V}$ be the module of derivations of $S$ to itself

$\operatorname{Der}_{V}=\{\theta: S \rightarrow S \mid \theta$ is $\mathbb{R}$-linear and $\theta(f g)=f \theta(g)+g \theta(f)$ for any $f, g \in S\}$.

The derivation module $D\left(\mathcal{S}_{\ell}\right)$ is defined by

$$
\begin{aligned}
D\left(\mathcal{S}_{\ell}\right)=\left\{\theta \in \operatorname{Der}_{V} \mid \theta(\alpha) \text { is divisible by } \alpha\right. \\
\text { and } \left.\theta(\alpha-z) \text { is divisible by } \alpha-z \text { for any } \alpha \in \Phi_{+}\right\} .
\end{aligned}
$$

In the present paper, for the first time, we construct an explicit basis for the derivation module $D\left(\mathcal{S}_{\ell}\right)$. The most important ingredients of our recipe are the Bernoulli polynomials $B_{k}(x)$ and their relatives $B_{p, q}(x)$ in Definition 2.2. The explicit costruction of our basis is in Definition 3.1.

One of the remarkable properties of the Shi arrangement is the fact that its number of chambers is equal to $(\ell+2)^{\ell}$. A good number of articles, including [9, 3, 4, 2, 13], study this intriguing property. Because of Zaslavsky's chamber counting formula [14], the property follows from the formula

$$
\pi\left(\mathcal{S}_{\ell}, t\right)=(1+t)(1+(\ell+1) t)^{\ell}
$$

for the Poincaré polynomial [5] of the cone $\mathcal{S}_{\ell}$. Ch. Athanasiadis proved that $D\left(\mathcal{S}_{\ell}\right)$ is a free $S$-module with exponents $(0,1, \ell+1, \ldots, \ell+1)$ in [2]. 
He consequently proved the formula above thanks to the factorization theorem in [11] which asserts that if the derivation module $D(\mathcal{A})$ is a free $S$ module with a basis $\theta_{1}, \ldots, \theta_{\ell}$ then the Poincaré polynomial of $\mathcal{A}$ is equal to $\prod_{j=1}^{\ell}\left(1+\left(\operatorname{deg} \theta_{j}\right) t\right)$. His proof of the freeness in [2] uses the additiondeletion theorem [10]. Later M. Yoshinaga extended this result in [13] to the generalized Shi and Catalan arrangements and affirmatively settled the Edelman-Reiner conjecture [3]. However, even in the case of Shi arrangements, no basis was constructed so far.

The organization of this paper is as follows: in Section 2, we will define the polynomials $B_{p, q}(x)$ which includes the Bernoulli polynomials. In Section 3, Theorem 3.5 proves that the derivations constructed in Definition 3.1 form a basis for the derivation module $D\left(\mathcal{S}_{\ell}\right)$.

\section{The Bernoulli polynomials and $B_{p, q}(x)$}

Let $B_{k}(x)$ denote the $k$-th Bernoulli polynomial. Let $B_{k}(0)=B_{k}$ denote the $k$-th Bernoulli number. The most important property of the Bernoulli polynomial in this paper is the following elementary formula (e.g., [1]):

\section{Theorem 2.1}

$$
B_{k}(x+1)-B_{k}(x)=k x^{k-1} .
$$

Definition 2.2 For $(p, q) \in\left(\mathbb{Z}_{\geq 0}\right)^{2}$, consider a polynomial $B_{p, q}(x)$ in $x$ satisfying the following two conditions:

1. $B_{p, q}(x+1)-B_{p, q}(x)=(x+1)^{p} x^{q}$,

2. $B_{p, q}(0)=0$.

It is easy to see that $B_{p, q}(x)$ is uniquely determined by these two conditions.

Example 2.3 (1) When $(p, q)=(0, q)$, we have

$$
B_{0, q}(x)=\frac{1}{q+1}\left\{B_{q+1}(x)-B_{q+1}\right\}
$$

because of Theorem 2.1.

(2) When $(p, q)=(p, 0)$, we obtain

$$
B_{p, 0}(x)=\frac{(-1)^{p+1}}{p+1}\left\{B_{p+1}(-x)-B_{p+1}\right\}=(-1)^{p+1} B_{0, p}(-x)
$$


because

$$
\begin{aligned}
& (-1)^{p+1} B_{0, p}(-x-1)-(-1)^{p+1} B_{0, p}(-x) \\
= & (-1)^{p}\left\{B_{0, p}(-x)-B_{0, p}(-x-1)\right\}=(-1)^{p}(-x-1)^{p}=(x+1)^{p} .
\end{aligned}
$$

(3) For a general $(p, q) \in\left(\mathbb{Z}_{\geq 0}\right)^{2}$, it easily follows from Theorem 2.1 that the polynomial has an expression in terms of the Bernoulli polynomials as

$$
B_{p, q}(x)=\sum_{i=0}^{p} \frac{1}{q+i+1}\left(\begin{array}{c}
p \\
i
\end{array}\right)\left\{B_{q+i+1}(x)-B_{q+i+1}\right\}=\sum_{i=0}^{p}\left(\begin{array}{l}
p \\
i
\end{array}\right) B_{0, q+i}(x) .
$$

For example, $B_{1,1}(x)=B_{0,1}(x)+B_{0,2}(x)=\frac{1}{3}\left(x^{3}-x\right)$.

Note that the polynomial $B_{p, q}(x)$ is a polynomial of degree $p+q+1$. The homogenization $\bar{B}_{p, q}(x, z)$ of $B_{p, q}(x)$ is defined by

$$
\bar{B}_{p, q}(x, z):=z^{p+q+1} B_{p, q}\left(\frac{x}{z}\right) .
$$

\section{A basis construction}

Let $1 \leq j \leq \ell$. Define

$$
I_{1}=\left\{x_{1}, x_{2}, \ldots, x_{j-1}\right\}, I_{2}=\left\{x_{j+2}, x_{j+3}, \ldots, x_{\ell+1}\right\} .
$$

Let $\sigma_{k}^{(s)}$ denote the elementary symmetric function in the variables in $I_{s}$ of degree $k\left(s=1,2, k \in \mathbb{Z}_{\geq 0}\right)$. Recall the homogeneous polynomials $\bar{B}_{p, q}(x, z)$ of degree $p+q+1$ defined at the end of the previous section.

Definition 3.1 Let $\partial_{i}(1 \leq i \leq \ell+1)$ and $\partial_{z}$ denote $\partial / \partial x_{i}$ and $\partial / \partial z$ respectively. Define homogeneous derivations

$$
\eta_{1}:=\sum_{i=1}^{\ell+1} \partial_{i} \in D\left(\mathcal{S}_{\ell}\right), \quad \eta_{2}:=z \partial_{z}+\sum_{i=1}^{\ell+1} x_{i} \partial_{i} \in D\left(\mathcal{S}_{\ell}\right),
$$

and

$$
\varphi_{j}:=\left(x_{j}-x_{j+1}-z\right) \sum_{i=1}^{\ell+1} \sum_{\substack{0 \leq k_{1} \leq j-1 \\ 0 \leq k_{2} \leq \ell-j}}(-1)^{k_{1}+k_{2}} \sigma_{j-1-k_{1}}^{(1)} \sigma_{\ell-j-k_{2}}^{(2)} \bar{B}_{k_{1}, k_{2}}\left(x_{i}, z\right) \partial_{i}
$$

for $1 \leq j \leq \ell$. 
We will prove that the derivations $\eta_{1}, \eta_{2}$, and $\varphi_{1}, \ldots, \varphi_{\ell}$ form a basis for $D\left(\mathcal{S}_{\ell}\right)$. First we will verify the following Proposition:

Proposition 3.2 The derivations $\varphi_{j}(1 \leq j \leq \ell)$ belong to the module $D\left(\mathcal{S}_{\ell}\right)$.

proof. We first have

$$
\begin{aligned}
\varphi_{j}\left(x_{p}-x_{q}\right)= & \left(x_{j}-x_{j+1}-z\right) \\
& \sum_{\substack{0 \leq k_{1} \leq j-1 \\
0 \leq k_{2} \leq \ell-j}}(-1)^{k_{1}+k_{2}} \sigma_{j-1-k_{1}}^{(1)} \sigma_{\ell-j-k_{2}}^{(2)}\left\{\bar{B}_{k_{1}, k_{2}}\left(x_{p}, z\right)-\bar{B}_{k_{1}, k_{2}}\left(x_{q}, z\right)\right\} .
\end{aligned}
$$

Since the right hand side equals zero if we set $x_{p}=x_{q}$, we may conclude that $\varphi_{j}\left(x_{p}-x_{q}\right)$ is divisible by $x_{p}-x_{q}$ for all pairs $(p, q)$ with $1 \leq p<q \leq \ell+1$.

The congruent notation $\equiv$ in the following calculation is modulo the ideal $\left(x_{p}-x_{q}-z\right)$ :

$$
\begin{aligned}
& \varphi_{j}\left(x_{p}-x_{q}-z\right) \\
\equiv & \left(x_{j}-x_{j+1}-z\right) \\
& \sum_{\substack{0 \leq k_{1} \leq j-1 \\
0 \leq k_{2} \leq \ell-j}}(-1)^{k_{1}+k_{2}} \sigma_{j-1-k_{1}}^{(1)} \sigma_{\ell-j-k_{2}}^{(2)}\left\{\bar{B}_{k_{1}, k_{2}}\left(x_{p}, x_{p}-x_{q}\right)-\bar{B}_{k_{1}, k_{2}}\left(x_{q}, x_{p}-x_{q}\right)\right\} \\
= & \left(x_{j}-x_{j+1}-z\right) \sum_{\substack{0 \leq k_{1} \leq j-1 \\
0 \leq k_{2} \leq \ell-j}}(-1)^{k_{1}+k_{2}} \sigma_{j-1-k_{1}}^{(1)} \sigma_{\ell-j-k_{2}}^{(2)} \\
= & \left(x_{j}-x_{j+1}-z\right)
\end{aligned}
$$$$
\sum_{\substack{0 \leq k_{1} \leq j-1 \\ 0 \leq k_{2} \leq \ell-j}}(-1)^{k_{1}+k_{2}} \sigma_{j-1-k_{1}}^{(1)} \sigma_{\ell-j-k_{2}}^{(2)}\left(x_{p}-x_{q}\right)^{k_{1}+k_{2}+1}\left(\frac{x_{p}}{x_{p}-x_{q}}\right)^{k_{1}}\left(\frac{x_{q}}{x_{p}-x_{q}}\right)^{k_{2}}
$$$$
=\left(x_{j}-x_{j+1}-z\right)\left(x_{p}-x_{q}\right) \sum_{\substack{0 \leq k_{1} \leq j-1 \\ 0 \leq k_{2} \leq \ell-j}}(-1)^{k_{1}+k_{2}} \sigma_{j-1-k_{1}}^{(1)} \sigma_{\ell-j-k_{2}}^{(2)} x_{p}^{k_{1}} x_{q}^{k_{2}}
$$$$
=\left(x_{j}-x_{j+1}-z\right)\left(x_{p}-x_{q}\right) \sum_{k_{1}=0}^{j-1} \sigma_{j-1-k_{1}}^{(1)}\left(-x_{p}\right)^{k_{1}} \sum_{k_{2}=0}^{\ell-j} \sigma_{\ell-j-k_{2}}^{(2)}\left(-x_{q}\right)^{k_{2}}
$$$$
=\left(x_{j}-x_{j+1}-z\right)\left(x_{p}-x_{q}\right) \prod_{s=1}^{j-1}\left(x_{s}-x_{p}\right) \prod_{s=j+2}^{\ell+1}\left(x_{s}-x_{q}\right) \equiv 0
$$

for all pairs $(p, q)$ with $1 \leq p<q \leq \ell+1$. 
Lemma 3.3 Suppose $\ell \geq 1$. Let $N$ be the $\ell \times \ell$-matrix whose $(i, j)$-entry is equal to the elementary symmetric function of degree $\ell-i$ in the variables $x_{1}, \ldots, x_{j-1}, x_{j+2}, \ldots, x_{\ell+1}$. Then

$$
\operatorname{det} N=(-1)^{\ell(\ell-1) / 2} \prod_{\substack{1 \leq p<q \leq \ell \\ q-p>1}}\left(x_{p}-x_{q}\right) .
$$

proof. Note that we have the equality

$$
\begin{aligned}
& {\left[\begin{array}{l}
1-x_{p} \\
\left.\left(-x_{p}\right)^{2} \ldots\left(-x_{p}\right)^{\ell-2}\left(-x_{p}\right)^{\ell-1}\right] N
\end{array}\right.} \\
&=\left[\prod_{\substack{1 \leq s \leq \ell+1 \\
s \notin\{1,2\}}}\left(x_{s}-x_{p}\right) \prod_{\substack{1 \leq s \leq \ell+1 \\
s \notin\{2,3\}}}\left(x_{s}-x_{p}\right) \ldots \prod_{\substack{1 \leq s \leq \ell+1 \\
s \notin\{\ell-1, \ell\}}}\left(x_{s}-x_{p}\right) \prod_{\substack{1 \leq s \leq \ell+1 \\
s \notin \ell \ell, \ell+1\}}}\left(x_{s}-x_{p}\right)\right]
\end{aligned}
$$

for any $1 \leq p \leq \ell$. Suppose that

$$
1 \leq p<q \leq \ell+1, q-p>1 .
$$

Set $x_{p}=x_{q}$ in $N$, and we get $N_{p q}$. Then we may conclude that

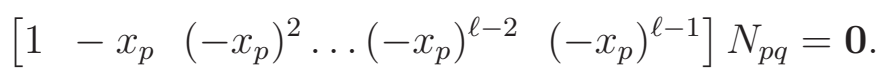

This implies that $\operatorname{det} N_{p q}=0$ and that $\operatorname{det} N$ is divisible by $x_{p}-x_{q}$. Since

$$
\operatorname{deg}(\operatorname{det} N)=\ell(\ell-1) / 2=\operatorname{deg} \prod_{\substack{1 \leq p<q \leq \ell+1 \\ q-p>1}}\left(x_{p}-x_{q}\right)
$$

there exists a constant $C$ such that

$$
\operatorname{det} N=C(-1)^{\ell(\ell-1) / 2} \prod_{\substack{1 \leq p<q \leq \ell+1 \\ q-p>1}}\left(x_{p}-x_{q}\right)=C \prod_{\substack{1 \leq p<q \leq \ell+1 \\ q-p>1}}\left(x_{q}-x_{p}\right) .
$$

By comparing the coefficients of $x_{3} x_{4}^{2} \ldots x_{\ell}^{\ell-2} x_{\ell+1}^{\ell-1}$ on both sides, we obtain $C=1$.

Proposition 3.4 The derivations $\eta_{1}, \eta_{2}, \varphi_{1}, \ldots, \varphi_{\ell}$ are linearly independent over $S$. 
proof. Set $z=0$ in $\varphi_{j}$ and we get $\phi_{j}$ as follows:

$$
\begin{aligned}
\phi_{j} & :=\left.\varphi_{j}\right|_{z=0}=\left(x_{j}-x_{j+1}\right) \sum_{i=1}^{\ell+1} \sum_{\substack{0 \leq k_{1} \leq j-1 \\
0 \leq k_{2} \leq \ell-j}} \frac{(-1)^{k_{1}+k_{2}}}{k_{1}+k_{2}+1} \sigma_{j-1-k_{1}}^{(1)} \sigma_{\ell-j-k_{2}}^{(2)} x_{i}^{k_{1}+k_{2}+1} \partial_{i} \\
& =\left(x_{j}-x_{j+1}\right) \sum_{k=1}^{\ell} \frac{(-1)^{k-1}}{k}\left(\sum_{\substack{k_{1}+k_{2}+1=k \\
0 \leq k_{1} \leq j-1 \\
0 \leq k_{2} \leq \ell-j}} \sigma_{j-1-k_{1}}^{(1)} \sigma_{\ell-j-k_{2}}^{(2)}\right) \sum_{i=1}^{\ell+1} x_{i}^{k} \partial_{i} \\
& =\left(x_{j}-x_{j+1}\right) \sum_{k=1}^{\ell} \frac{(-1)^{k-1}}{k} \sigma_{\ell-k}\left(x_{1}, \ldots, x_{j-1}, x_{j+2}, \ldots, x_{\ell+1}\right) \sum_{i=1}^{\ell+1} x_{i}^{k} \partial_{i} .
\end{aligned}
$$

Here $\sigma_{\ell-i}\left(x_{1}, \ldots, x_{j-1}, x_{j+2}, \ldots, x_{\ell+1}\right)$ stands for the elementary symmetric function of degree $\ell-i$ in the variables $x_{1}, \ldots, x_{j-1}, x_{j+2}, \ldots, x_{\ell+1}$. This is equal to the $(i, j)$-entry $N_{i j}$ of the matrix $N$ in Lemma 3.3. Thus we have

$$
\phi_{j}\left(x_{i}\right)=\left(x_{j}-x_{j+1}\right) \sum_{k=1}^{\ell} \frac{(-1)^{k-1}}{k} x_{i}^{k} N_{k j} .
$$

Define two $(\ell+1) \times(\ell+1)$-diagonal matrices $D_{1}$ and $D_{2}$ by

$$
\begin{aligned}
D_{1} & :=[1] \oplus[1] \oplus\left[(-1)^{1} / 2\right] \oplus\left[(-1)^{2} / 3\right] \oplus \cdots \oplus\left[(-1)^{\ell-1} / \ell\right], \\
D_{2} & :=[1] \oplus\left[x_{1}-x_{2}\right] \oplus\left[x_{2}-x_{3}\right] \oplus \cdots \oplus\left[x_{\ell}-x_{\ell+1}\right]
\end{aligned}
$$

where $\oplus$ stands for the direct sum of matrices. Also define two $(\ell+1) \times(\ell+1)$ matrices $\tilde{N}$ and $M$ by

$$
\tilde{N}:=[1] \oplus N, \quad M:=\left[x_{i}^{j-1}\right]_{1 \leq i \leq \ell+1,1 \leq j \leq \ell+1} .
$$

From (3.1) we obtain

$$
P:=\left[\begin{array}{cccc}
1 & \phi_{1}\left(x_{1}\right) & \ldots & \phi_{\ell}\left(x_{1}\right) \\
1 & \phi_{1}\left(x_{2}\right) & \ldots & \phi_{\ell}\left(x_{2}\right) \\
1 & \phi_{1}\left(x_{3}\right) & \ldots & \phi_{\ell}\left(x_{3}\right) \\
\cdot & \cdot & \ldots & \cdot \\
\cdot & \cdot & \ldots & \cdot \\
1 & \phi_{1}\left(x_{\ell+1}\right) & \ldots & \phi_{\ell}\left(x_{\ell+1}\right)
\end{array}\right]=M D_{1} \tilde{N} D_{2}
$$


Thus, by applying the Vandermonde determinant formula and Lemma 3.3, we deduce

$$
\begin{aligned}
\operatorname{det} P & =(\operatorname{det} M)\left(\operatorname{det} D_{1}\right)(\operatorname{det} \tilde{N})\left(\operatorname{det} D_{2}\right) \\
& =\left(\prod_{1 \leq p<q \leq \ell+1}\left(x_{q}-x_{p}\right)\right)\left(\frac{(-1)^{\ell(\ell-1) / 2}}{\ell !}\right)(\operatorname{det} N) \prod_{1 \leq p \leq \ell}\left(x_{p}-x_{p+1}\right) \\
& =\left(\frac{(-1)^{\ell(\ell+1) / 2}}{\ell !}\right) \prod_{1 \leq p<q \leq \ell+1}\left(x_{p}-x_{q}\right)^{2} \neq 0 .
\end{aligned}
$$

Thus $\eta_{1}, \phi_{1}, \ldots, \phi_{\ell}$ are linearly independent. This implies that $\eta_{1}, \varphi_{1}, \ldots, \varphi_{\ell}$ are linearly independent. Since $\eta_{2}(z)=z$ and $\eta_{1}(z)=\varphi_{1}(z)=\cdots=\varphi_{\ell}(z)=$ 0 , we finally conclude that $\eta_{1}, \eta_{2}, \varphi_{1}, \ldots, \varphi_{\ell}$ are linearly independent.

Remark. The derivations $\phi_{1}, \ldots, \phi_{\ell}$ are a basis for the derivation module of the double Coxeter arrangement of the type $A_{\ell}$ studied in [8] (cf. [12]).

Theorem 3.5 The derivations $\eta_{1}, \eta_{2}, \varphi_{1}, \ldots, \varphi_{\ell}$ form a basis for $D\left(\mathcal{S}_{\ell}\right)$.

proof. We may apply Saito's criterion [6] [5, Theorem 4.23] thanks to Propositions 3.2 and 3.4 because

$$
\operatorname{deg} \eta_{1}+\operatorname{deg} \eta_{2}+\sum_{j=1}^{\ell} \operatorname{deg} \varphi_{j}=1+\ell(\ell+1)=\left|\mathcal{S}_{\ell}\right| .
$$

Remark. The Bernoulli polynomials explicitly appear in the first derivation $\varphi_{1}$ and the last one $\varphi_{\ell}$ because of Example 2.3 (1) and (2):

$$
\begin{aligned}
\varphi_{1} & =\left(x_{1}-x_{2}-z\right) \sum_{i=1}^{\ell+1} \sum_{k_{2}=0}^{\ell-1}(-1)^{k_{2}} \sigma_{\ell-1-k_{2}}^{(2)} \bar{B}_{0, k_{2}}\left(x_{i}, z\right) \partial_{i} \\
& =\left(x_{1}-x_{2}-z\right) \sum_{i=1}^{\ell+1} \sum_{k=1}^{\ell} \frac{(-1)^{k-1}}{k} \sigma_{\ell-k}^{(2)} z^{k}\left(B_{k}\left(x_{i} / z\right)-B_{k}\right) \partial_{i},
\end{aligned}
$$

and

$$
\begin{aligned}
\varphi_{\ell} & =\left(x_{\ell}-x_{\ell+1}-z\right) \sum_{i=1}^{\ell+1} \sum_{k_{1}=0}^{\ell-1}(-1)^{k_{1}} \sigma_{\ell-1-k_{1}}^{(1)} \bar{B}_{k_{1}, 0}\left(x_{i}, z\right) \partial_{i} \\
& =\left(x_{\ell}-x_{\ell+1}-z\right) \sum_{i=1}^{\ell+1} \sum_{k=1}^{\ell} \frac{(-1)^{k-1}}{k} \sigma_{\ell-k}^{(1)}(-z)^{k}\left(B_{k}\left(-x_{i} / z\right)-B_{k}\right) \partial_{i} .
\end{aligned}
$$

Here $\sigma_{d}^{(1)}$ and $\sigma_{d}^{(2)}$ are the elementary symmetric functions of degree $d$ in the variables $x_{1}, \ldots, x_{\ell-1}$ and $x_{3}, \ldots, x_{\ell+1}$ respectively. 
Example 3.6 For $A_{3}$, we have

$$
\begin{aligned}
\eta_{1}= & \partial_{1}+\partial_{2}+\partial_{3}+\partial_{4}, \quad \eta_{2}=x_{1} \partial_{1}+x_{2} \partial_{2}+x_{3} \partial_{3}+x_{4} \partial_{4}+z \partial_{z}, \\
\varphi_{1}= & x_{1}\left(x_{1}-x_{2}-z\right)\left\{x_{3} x_{4}-\frac{1}{2}\left(x_{3}+x_{4}\right)\left(x_{1}-z\right)+\frac{1}{3}\left(x_{1}^{2}-\frac{3}{2} x_{1} z+\frac{1}{2} z^{2}\right)\right\} \partial_{1} \\
& +x_{2}\left(x_{1}-x_{2}-z\right)\left\{x_{3} x_{4}-\frac{1}{2}\left(x_{3}+x_{4}\right)\left(x_{2}-z\right)+\frac{1}{3}\left(x_{2}^{2}-\frac{3}{2} x_{2} z+\frac{1}{2} z^{2}\right)\right\} \partial_{2} \\
& -\frac{1}{6} x_{3}\left(x_{1}-x_{2}-z\right)\left(x_{3}+z\right)\left(x_{3}-3 x_{4}-z\right) \partial_{3} \\
& -\frac{1}{6} x_{4}\left(x_{1}-x_{2}-z\right)\left(x_{4}+z\right)\left(x_{4}-3 x_{3}-z\right) \partial_{4}, \\
\varphi_{2}= & -\frac{1}{6} x_{1}\left(x_{2}-x_{3}-z\right)\left(x_{1}-z\right)\left(x_{1}-3 x_{4}-2 z\right) \partial_{1} \\
& +x_{2}\left(x_{2}-x_{3}-z\right)\left\{x_{1} x_{4}-\frac{1}{2} x_{1}\left(x_{2}-z\right)-\frac{1}{2} x_{4}\left(x_{2}+z\right)+\frac{1}{3}\left(x_{2}^{2}-z^{2}\right)\right\} \partial_{2} \\
& +x_{3}\left(x_{2}-x_{3}-z\right)\left\{x_{1} x_{4}-\frac{1}{2} x_{1}\left(x_{3}-z\right)-\frac{1}{2} x_{4}\left(x_{3}+z\right)+\frac{1}{3}\left(x_{3}^{2}-z^{2}\right)\right\} \partial_{3} \\
& +\frac{1}{6} x_{4}\left(x_{2}-x_{3}-z\right)\left(x_{4}+z\right)\left(3 x_{1}-x_{4}-2 z\right) \partial_{4}, \\
= & -\frac{1}{6} x_{1}\left(x_{3}-x_{4}-z\right)\left(x_{1}-z\right)\left(x_{1}-3 x_{2}+z\right) \partial_{1} \\
& -\frac{1}{6} x_{2}\left(x_{3}-x_{4}-z\right)\left(x_{2}-z\right)\left(x_{2}-3 x_{1}+z\right) \partial_{2} \\
+ & x_{3}\left(x_{3}-x_{4}-z\right)\left\{x_{1} x_{2}-\frac{1}{2}\left(x_{1}+x_{2}\right)\left(x_{3}+z\right)+\frac{1}{3}\left(x_{3}^{2}+\frac{3}{2} x_{3} z+\frac{1}{2} z^{2}\right)\right\} \partial_{3} \\
+ & x_{4}\left(x_{3}-x_{4}-z\right)\left\{x_{1} x_{2}-\frac{1}{2}\left(x_{1}+x_{2}\right)\left(x_{4}+z\right)+\frac{1}{3}\left(x_{4}^{2}+\frac{3}{2} x_{4} z+\frac{1}{2} z^{2}\right)\right\} \partial_{4} .
\end{aligned}
$$

Problem. Construct a basis for the derivation module $D(\mathcal{A})$ when $\mathcal{A}$ is the cone over the generalized Shi arrangement of one of the other types: $B_{\ell}, C_{\ell}, D_{\ell}, E_{6}, E_{7}, E_{8}, F_{4}$ and $G_{2}$. It has been known that the derivation modules are free modules for these cases by M. Yoshinaga [13]. It seems interesting to learn what kind of polynomials plays the role of the Bernoulli polynomials in the case of the type $A_{\ell}$.

\section{References}

[1] T. M. Apostol, Introduction to analytic number theory. Undergraduate Texts in Mathematics, Springer-Verlag, New York-Heidelberg, 1976. 
[2] Ch. Athanasiadis, On free deformations of the braid arrangement. European J. Combin. 19 (1998), 7-18.

[3] P. H. Edelman and V. Reiner, Free arrangements and rhombic tilings. Discrete Comp. Geom. 15 (1996), 307-340.

[4] P. Headley, On a family of hyperplane arrangements related to the affine Weyl groups. J. Algebraic Combin. 6 (1997), 331-338.

[5] P. Orlik and H. Terao, Arrangements of hyperplanes. Grundlehren der Mathematischen Wissenschaften, 300. Springer-Verlag, Berlin, 1992.

[6] K. Saito, Theory of logarithmic differential forms and logarithmic vector fields. J. Fac. Sci. Univ. Tokyo Sect. IA Math. 27 (1980), 265-291.

[7] J.-Y. Shi, The Kazhdan-Lusztig cells in certain affine Weyl groups. Lecture Notes in Math., 1179, Springer-Verlag, 1986.

[8] L. Solomon and H. Terao, The double Coxeter arrangements. Comment. Math. Helv. 73 (1998), 237-258.

[9] R. P. Stanley, Hyperplane arrangements, interval orders and trees. Proc. Natl. Acad. Sci., 93 (1996), 2620-2625.

[10] H. Terao, Arrangements of hyperplanes and their freeness I, II. J. Fac. Sci. Univ. Tokyo 27 (1980), 293-320.

[11] H. Terao, Generalized exponents of a free arrangement of hyperplanes and Shephard-Todd-Brieskorn formula. Invent. math. 63 (1981), 159179.

[12] H. Terao, Multiderivations of Coxeter arrangements. Invent. Math. 148 (2002), 659-674.

[13] M. Yoshinaga, Characterization of a free arrangement and conjecture of Edelman and Reiner. Invent. Math. 157 (2004), 449-454.

[14] T. Zaslavsky, Facing up to arrangements: Face-count formulas for partitions of space by hyperplanes, Mem. Amer. Math. Soc. 154 (1975). 